\title{
Are Patients at Nutritional Risk More Prone to Complications after Major Urological Surgery?
}

\author{
Yannick Cerantola,* Massimo Valerio, Martin Hubner, Katia Iglesias, \\ Laurent Vaucher and Patrice Jichlinski \\ From the Urology (YC, MV, LV, PJ) and Visceral Surgery (MH) Services and Center for Clinical Research (KI), University Hospital \\ Centre Hospitalier Universitaire Vaudois, Lausanne, Switzerland
}

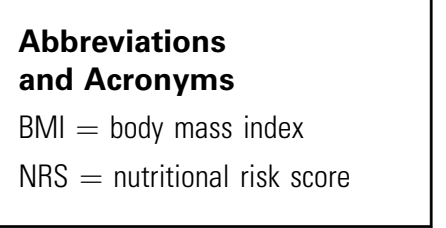

Accepted for publication June 27, 2013

Study received the institutional board and local ethical committee approval.

* Correspondence: Service d'Urologie, Centre Hospitalier Universitaire Vaudois, Bugnon 46, 1011 Lausanne-CHUV, Switzerland (telephone: +41795568707; FAX: +41213142992; e-mail: yannick.cerantola@chuv.ch.

Purpose: The nutritional risk score is a recommended screening tool for malnutrition. While a nutritional risk score of 3 or greater predicts adverse outcomes after digestive surgery, to our knowledge its predictive value for morbidity after urological interventions is unknown. We determined whether urological patients at nutritional risk are at higher risk for complications after major surgery than patients not at nutritional risk.

Materials and Methods: We performed a prospective observational study in consecutive patients undergoing major surgery. A priori sample calculation resulted in a study cohort of 220 patients. Interim analysis was planned after 110 patients. The nutritional risk score was assessed preoperatively by a specialized study nurse. Nutritional care was standardized in all patients. Postoperative complications were defined previously using the standardized Dindo-Clavien classification. The primary end point was 30-day morbidity. Univariate and multivariate analysis was performed to identify predictors of complications.

Results: The study was discontinued due to significant results after interim analysis. A total of 125 patients were included in analysis from June 2011 to June 2012 and 15 were excluded because of incomplete data. Of 51 patients at nutritional risk 38 (74\%) presented with at least 1 complication compared to 28 of 59 controls $(47 \%)$. Patients at nutritional risk were at threefold risk for complications on univariate and multivariate analysis (OR 3.3, 95\% CI 1.3-8.0). Cystectomy was the only other predictor of morbidity (OR 10, 95\% CI 2-48).

Conclusions: Patients at nutritional risk are more prone to complications after major urological procedures. Whether this increased morbidity can be reversed by perioperative nutritional support should be studied.

Key Words: urology, surgery, malnutrition, complications, risk

MaLnUtRition affects about $40 \%$ of hospitalized patients ${ }^{1}$ and is associated with increased mortality, morbidity and hospital stay after digestive surgery. ${ }^{2}$ Since malnutrition represents the most important modifiable risk factor, efforts have been made to develop clinical screening tools. $^{3}$ The European Society for Parenteral and Enteral Nutrition
(ESPEN), and American Society for Parenteral and Enteral Nutrition (ASPEN) recommend using the prospectively validated NRS (fig. 1). ${ }^{1,4}$ The score is composed of 3 components, including nutritional status, disease/intervention severity and patient age. By attributing points to low BMI, decreased oral food intake or weight loss ( 0 to 3 points), increased 


\begin{tabular}{|c|c|c|c|c|}
\hline & Nutritional Status & Disease/Surgery Severity & Age & \\
\hline 0 & Normal & Normal & $<70 \mathrm{yr}$ & \\
\hline 1 & $\begin{array}{l}\text { Weight loss }>5 \% / 3 \text { mo OR } \\
\text { Food intake }<75 \%\end{array}$ & $\begin{array}{l}\text { Chronic disease, hip fracture, } \\
\text { cancer, minor surgery, ... }\end{array}$ & $\geq 70 \mathrm{yr}$ & \\
\hline 2 & $\begin{array}{l}\text { Weight loss }>5 \% / 2 \text { mo OR } \\
\text { Food intake }<50 \% \text { OR } \\
\text { BMI 18.5-20.5 }\end{array}$ & $\begin{array}{l}\text { Major surgery, myocardial } \\
\text { infarction, pneumonia, } \\
\text { lymphoma, leukemia, ... }\end{array}$ & & \\
\hline 3 & $\begin{array}{l}\text { Weight loss }>5 \% / 1 \mathrm{mo} \\
\text { (or }>15 \% / 3 \mathrm{mo}) \text { OR } \\
\text { Food intake }<25 \% \text { OR } \\
\text { BMI }<18.5\end{array}$ & $\begin{array}{l}\text { Head trauma, transplantation, } \\
\text { intensive care patients, ... }\end{array}$ & & NRS \\
\hline & + & + & & $=$ \\
\hline
\end{tabular}

Figure 1. NRS is calculated by adding highest value from each column with range of 0 to 7 . Patients with NRS 3 or greater are considered at risk for malnutrition. Weight loss is estimated as percent of total body mass. Food intake is evaluated by asking patient, "Within last week, were you able to eat whole meal/three-quarters of your meal/half of your meal/quarter of your meal?" $\mathrm{BMI}$ is shown in $\mathrm{kg} / \mathrm{m}^{2}$. mo, months. $y r$, years.

surgical stress ( 0 to 3 points) and age greater than 70 years (1 point) the NRS allows for the identification of patients at risk for malnutrition (NRS 3 or greater). ${ }^{1}$

While the risk of malnutrition has been largely studied for digestive surgery, ${ }^{2,5,6}$ little is known about its impact on postoperative morbidity in urology. Karl et al recently reported that up to $33 \%$ of urological patients who underwent open surgery at a urology department in Germany were at risk for malnutrition (NRS 3 or greater). ${ }^{7}$ In addition, recently 2 groups from the United States retrospectively evaluated the effect of preoperative nutritional deficiency on perioperative mortality and overall survival in patients undergoing oncological radical cystectomy or nephrectomy. 8,9 Nutrition deficiency was a strong independent factor for mortality in each study. Unfortunately, no data were available on morbidity.

Because urological surgery differs from digestive surgery in many points, the results of the latter cannot be extrapolated by implication. There is an urgent need to clarify the role of malnutrition in urology to properly use this information for preoperative counseling and risk stratifying, and also investigate whether perioperative nutritional intervention could positively affect the outcome, as shown for digestive surgery. ${ }^{10,11}$ To our knowledge no group has prospectively evaluated the impact of nutritional risk on morbidity after urological surgery. This study aims to fill this gap.

\section{MATERIALS AND METHODS}

The current study was designed as a prospective, single center observational study. We tested the hypothesis that patients at nutritional risk (NRS 3 or greater) would be exposed to increased postoperative morbidity. The second aim was to identify other possible predictors of complications, namely the Charlson comorbidity index, American Society of Anesthesiologists (ASA) score, age, sex, BMI, previous abdominal surgery, smoking habits, oncological indication, anemia and hypoalbuminemia.

After receiving approval from the institutional board and local ethical committee (No. 34/11, April 27, 2011), consecutive patients treated with major elective surgery were prospectively included in analysis. Since no standard definition of major surgery exists, it was defined as any open surgery performed with the patient under general anesthesia that was planned to last more than 2 hours. Patients undergoing surgery for stone disease were excluded from study to avoid any potential infectious bias. Patients undergoing laparoscopic or robotic surgery were also excluded.

After providing written informed consent patients were enrolled in the study by the operating urologist about 3 weeks preoperatively. Notably, extended nutritional screening and treatment were not yet part of standard perioperative management in our department at the time of this study. Therefore, the few patients who received supplementation preoperatively were not included in this study to avoid possible bias related to preoperative nutritional support.

All patients were admitted to the hospital the day before surgery. Weight and height were measured, and a blood sample was obtained to determine hemoglobin and albumin. A specialized study nurse then determined the NRS and calculated the modified Charlson comorbidity index. Demographic information, including age, gender, BMI, etc, were recorded in a specifically designed Excel ${ }^{\circledR}$ 2007 spreadsheet. All complications were defined before the study began and previously reported. ${ }^{12}$ Maximal length of stay was defined for each procedure. Postoperative nutritional support was standardized and all patients received 2 daily oral Ensure ${ }^{\circledR}$ Plus nutritional supplements in the first 3 days postoperatively, in addition to the standardized diet.

Complications were documented perioperatively by our specialized study nurse, who supervised resident 
reporting of complications. All patients were discussed at our daily grand round and complications were noted. Moreover, the final report at hospital discharge was reread by the study nurse to ensure that no supplementary complications had been missed.

All patients attended an assessment visit 1 month after surgery. They were asked about any complications that could have developed at home. Those complications were reported on a standardized case report form and collected by the study nurse. The modified Dindo-Clavien classification was used to stratify complications (see Appendix). ${ }^{13}$

A sample size calculation was run on certain information. A 50\% 30-day postoperative complication rate was assumed for patients at risk and a $25 \%$ rate was assumed for well nourished patients. A $40 \%$ prevalence of nutritional risk was expected in hospitalized patients treated with surgery. By adopting $90 \%$ power and a 5\% 2-sided type I error, and considering interim analysis the calculated sample size was 220 patients. Interim analysis was planned for after half the sample total size was recruited. A stopping rule was defined for statistically significant benefit.

Descriptive statistics are reported as the median and $\mathrm{IQR}$ or the mean $\pm \mathrm{SD}$ for continuous variables depending on the distribution, and the frequency and percent for categorical variables. To compare the 2 NRS groups we used the independent t-test or Mann-Whitney $U$ test for continuous variables, and the Pearson chi-square test for categorical variables. To examine risk factors for complications we created univariate and multivariate logistic regression models to analyze the impact of age, gender, BMI, history of smoking or abdominal surgery, ASA score, anemia, albumin, surgical indication, Charlson comorbidity index and NRS variables. A stepwise selection procedure was used. ${ }^{14}$ The OR on univariate analysis and the adjusted OR for multivariate analysis with the associated $95 \%$ CIs are reported for each explanatory variable. All analysis was done using Stata ${ }^{\circledR}$, release 12 with significance considered at $\alpha=5 \%$.

\section{RESULTS}

A total of 125 patients were prospectively included in the study from June 2011 to June 2012 (fig. 2). After excluding 15 patients due to incomplete data, including 8 with NRS less than 3 and 7 with NRS 3 or greater, 110 (26 women) were included in final analysis since interim analysis revealed a significantly worse outcome in patients at nutritional risk $(\mathrm{p}<0.005)$. A risk of malnutrition (NRS 3 or greater) was found in 51 patients $(46 \%)$. Mean age $\pm \mathrm{SD}$ was $63 \pm 14$ years. Mean BMI was $27 \pm 4 \mathrm{~kg} / \mathrm{m}^{2}$ and 31 patients $(28 \%)$ were obese (BMI greater than $30 \mathrm{~kg} / \mathrm{m}^{2}$ ). Of the patients $74 \%$ had an ASA score of 2 . Previous abdominal surgery had been done in $32 \%$ cases. Only $26 \%$ of patients were smokers. Anemia and hypoalbuminemia were found preoperatively in $25 \%$ and $21 \%$ of the prospective cohort, respectively. The mean albumin level was $43 \pm 6 \mathrm{gm} / \mathrm{l}$. Of the patients $53 \%$ had a Charlson comorbidity index of 0 .

Table 1 lists the characteristics of patients at nutritional risk (NRS 3 or greater) vs those not at
Power calculation

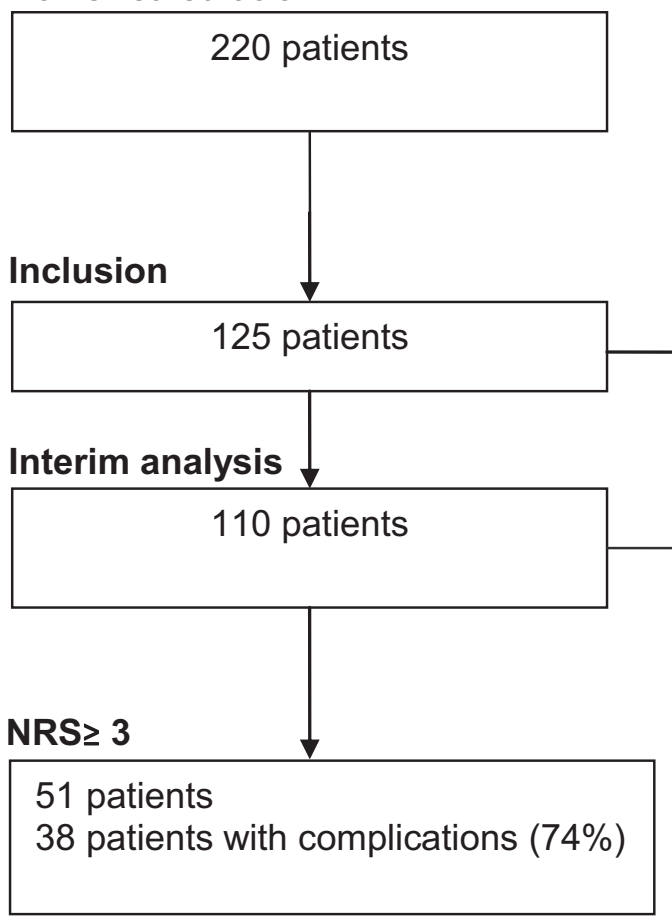

\section{Exclusion}

15 patients $(7 \mathrm{NRS} \geq 3$ and 8 $N R S<3$ ) because of incomplete data concerning main outcome 
Table 1. Patient characteristics

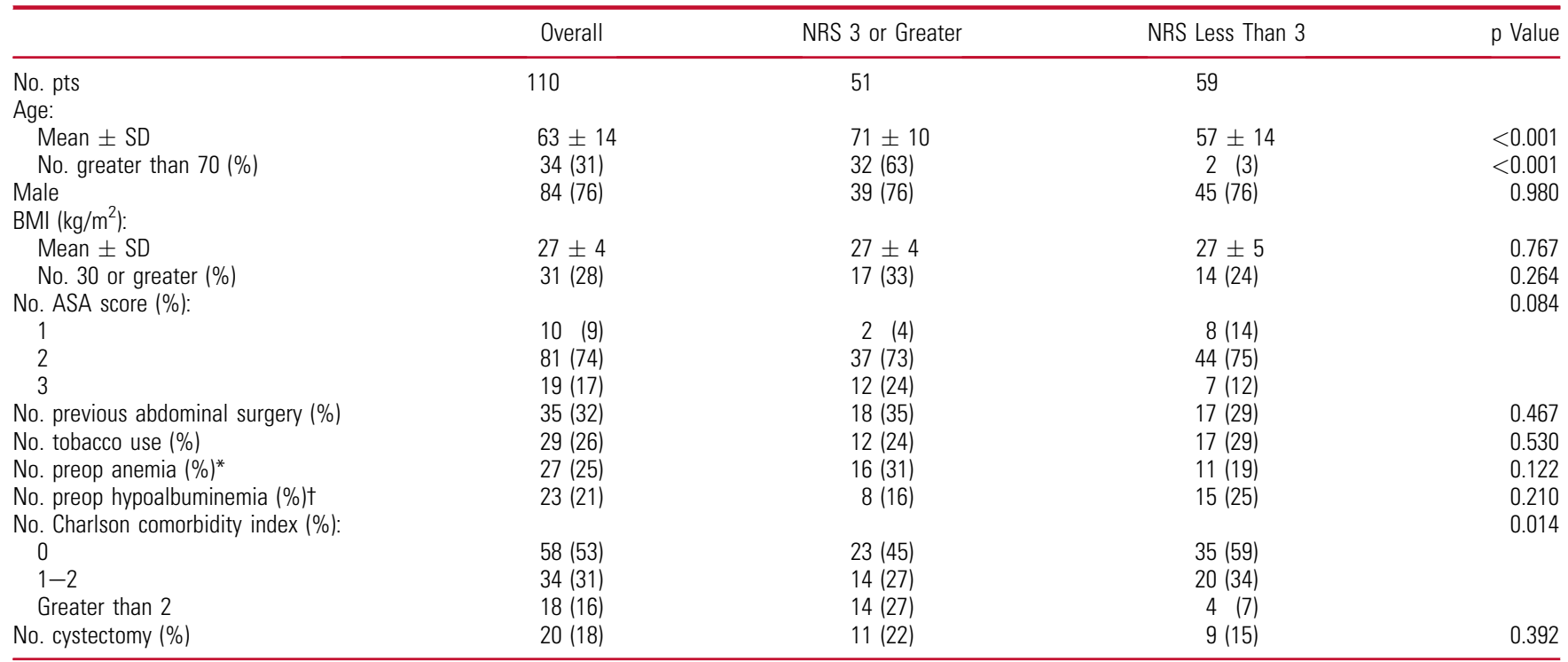

${ }^{*}$ Hematocrit less than $40 \%$ and less than $35 \%$ in male and female patients, respectively.

† Less than $35 \mathrm{gm} / \mathrm{l}$ albumin.

risk (NRS less than 3). Surgical interventions consisted of 46 prostatectomies, 41 retroperitoneal surgeries, 20 cystectomies and 3 functional operations.

No death occurred during the study period. A total of 66 patients $(60 \%)$ presented with at least 1 complication in the 30-day postoperative period. Major complications developed in 10 patients (table 2). Most complications were graded DindoClavien I or II with bleeding usually requiring blood transfusion and ileus occurring most frequently (25 and 10 cases, respectively). The complication rate in patients at nutritional risk was $75 \%$ (38 of 51) vs $47 \%$ (28 of 59) in those not at risk (OR 3.24, 95\% CI $1.44-7.28, \mathrm{p}=0.005$ ).

Univariate analysis revealed that cystectomy (OR 10.4, 95\% CI 2.14-50.83, p = 0.004) and a Charlson comorbidity index of greater than 0 (OR $2.47,95 \%$ CI $1.12-5.44, \mathrm{p}=0.025$ ) were also associated with postoperative complications. Other preoperative characteristics were not predictive, such as age, sex, BMI, previous abdominal surgery, smoking habits, ASA score, oncological indication, anemia and hypoalbuminemia.

On multivariate analysis only NRS 3 or greater (OR 3.27, 95\% CI 1.33-8.02, $\mathrm{p}=0.01$ ) and cystectomy (OR 9.66, 95\% CI 1.92-48.55, $\mathrm{p}=0.006$ ) were retained as significant independent predictors of complications.

\section{DISCUSSION}

The current study shows that patients at nutritional risk, as assessed by the NRS, are more prone to complications after major urological procedures. To our knowledge this is the first prospective study to link malnutrition with postoperative morbidity in urological patients undergoing major surgery.

Karl et al retrospectively correlated the risk of malnutrition to complications in a mixed (medical and surgical) urological population. ${ }^{15}$ of the 320 screened patients $68(21 \%)$ were identified to be at nutritional risk (NRS 3 or greater). Unfortunately,

Table 2. Major complications (Dindo-Clavien classification 3 or greater)

\begin{tabular}{|c|c|c|c|c|c|c|}
\hline Pt No. & Main Surgery & Oncological & Pt NRS & Complication & Treatment & $\begin{array}{l}\text { Dindo-Clavien } \\
\text { Classification }\end{array}$ \\
\hline 1 & Transvesical prostatectomy & No & 4 & Bleeding & Reoperation, hemostatic suturing & $3 b$ \\
\hline 2 & Nephrectomy & Yes & 2 & Pulmonary embolism & Catheter embolectomy & $3 a$ \\
\hline 3 & Partial nephrectomy & Yes & 2 & Bleeding & Reoperation, clot evacuation & $3 b$ \\
\hline 4 & Nephroureterectomy & Yes & 2 & Bleeding & Reoperation, packing, intensive care unit & $4 b$ \\
\hline 5 & Transvesical prostatectomy & No & 4 & Bleeding & Reoperation, hemostatic suturing & $3 b$ \\
\hline 6 & Radical prostatectomy & Yes & 3 & Bleeding & Embolization & $3 a$ \\
\hline 7 & Cystectomy + Bricker & Yes & 2 & Wound infection & Abscess drainage using general anesthesia & $3 b$ \\
\hline 8 & Radical prostatectomy & Yes & 3 & Lymphocele & $\begin{array}{l}\text { Percutaneous drainage under computerized } \\
\text { tomography guidance }\end{array}$ & $3 a$ \\
\hline 9 & Cystectomy + Bricker & Yes & 3 & Anastomotic (ileo-ileal) leak & Reanastomosis & $3 b$ \\
\hline 10 & Cystectomy + Studer & Yes & 3 & Anastomotic ureteral-neobladder stricture & Nephrostomy + antegrade balloon dilatation & 3a \\
\hline
\end{tabular}


complications were not predefined and Karl et al did not stratify patients by other potential confounders such as comorbidity. They concluded that the complication rate was higher in malnourished patients (6 of 68 vs 16 of 252) but the study was hampered by several methodological flaws.

In our study 51 patients (46\%) were at risk for malnutrition, greater than the $33 \%$ of urological patients treated with open surgery described by Karl et al. ${ }^{7}$ In contrast, Roth et al from Bern reported their experience with postoperative parenteral nutritional support in 157 patients undergoing radical cystectomy and found that a risk of malnutrition, defined as NRS 3 or greater, was present in $84(54 \%) .{ }^{16}$ This shows the wide variation of malnutrition in hospitalized patients upon admission and preoperatively. ${ }^{2,6,17}$ Factors such as decreased oral food intake, preexisting chronic disease, tumor cachexia and low socioeconomic status were identified as risk factors for malnutrition and contribute to the wide variation of its prevalence. ${ }^{6}$

At first glance the complication rate in our study seems higher than usually reported. We believe that this unusually high morbidity is related to the study design and the strict, standardized methodology of reporting complications. Our prospective study adheres to the criteria of Martin et $\mathrm{al}^{18}$ and Donat ${ }^{19}$ for reporting complications. Moreover, we used the Dindo-Clavien classification, which considers any deviation from the standard postoperative course as a complication. In the current study 20 patients (30\%) had grade I complications, which might have been underreported in less strict studies. Our study nurse also had a key role in prospectively recording complications. When this task has been left to residents, $80 \%$ of complications are not recorded even after extensive audit and teaching. ${ }^{20}$ This alarming statement reinforces the quality of our study and explains the high morbidity.

Despite improved surgical technique and anesthetic protocols surgery still elicits central nervous system mediated release of stress hormones and inflammatory mediators. ${ }^{21}$ This so-called surgical stress response ${ }^{22}$ causes catabolism of glycogen, fat and proteins, resulting in systemic release of glucose, and free fatty and amino acids. The anabolic state required for optimal patient rehabilitation, immune response and wound healing is not attained. ${ }^{3}$ Therefore, by placing the patient in a catabolic state before the surgical stress response occurs preoperative malnutrition might be an independent risk factor for increased morbidity and mortality after major urological surgery, as shown for digestive surgery. ${ }^{23,24}$

We used the NRS to assess the nutritional risk for several reasons. 1) It is the screening tool for malnutrition recommended by ESPEN and
ASPEN. ${ }^{1,4} 2$ ) It is easily implemented in clinical practice since it does not require further biological or anthropological parameters. 3) It was internally and externally validated, and correlates with other biological screening tools. ${ }^{25,26}$

However, in the current study NRS did not correlate with a biological measure since hypoalbuminemia was found in only $21 \%$ of patients. This might be explained by the relatively acute (within 0 to 4 weeks) onset of malnutrition in some patients. Indeed, albumin levels usually reflect nutritional status in the last month in relatively stable patients. Moreover, albumin is influenced by inflammation, age, muscle mass, hepatic failure, etc and, therefore, it is suboptimal. In the current series albumin levels did not correlate with age. Although prealbumin is known to be more sensitive for detecting early malnutrition and might have been useful in this study, we decided not to sample it to keep costs low. This can be considered a study limitation.

Another potential limitation of this study is population heterogeneity since patients treated with different type of major surgery were included and analyzed as a whole. Although we believe that this prospective cohort of patients is representative of most centers with a mixed case load, it would have been relevant to stratify patients by surgical procedure, eg cystectomy, prostatectomy and retroperitoneal surgery. However, because of the limited number of patients undergoing each type of procedure, subgroup analysis was not feasible. While complications might be procedure specific, all of these surgical interventions induce a common stress in the patient. Since to our knowledge no definition of major surgery is available in the literature, we used a straightforward definition including 3 criteria, namely open surgery, general anesthesia and operative time of about 2 hours.

In addition, a possible limitation that may have affected our results is the early interruption of the study before recruiting the total number of patients. Such interim analysis might have led to potential overestimation of the effect. Nevertheless, the data monitoring committee believed that it would be unethical to pursue the study while knowing that nutritional supplements could have a favorable impact on patients at nutritional risk.

While we acknowledge these possible limitations, we believe that this study has a number of important clinical implications for the urological community that should reinforce the implementation of NRS screening in daily clinical practice. 1) The prevalence of patients deemed to be at risk for malnutrition is high, ranging from $30 \%$ to $50 \%$ in different studies. 2) The NRS was the only preoperative score that could predict early morbidity after stratifying patients by other possible confounders. Other 
standardized and widely used tools, such as the Charlson comorbidity index and ASA score, were less useful than the NRS for predicting complications. 3) Nutritional status is at least partly a modifiable risk factor. To our knowledge whether preoperative nutritional support in patients at nutritional risk who are undergoing major urological surgery can improve postoperative outcomes remains unknown. However, preoperative oral nutritional supplementation, especially immunonutrition, ${ }^{11}$ can decrease the postoperative morbidity, length of hospital stay and costs of major gastrointestinal surgery. ${ }^{5,27,28}$ Well designed randomized, controlled trials specific to urological surgery might provide a definitive answer. Finally, the NRS is an easy, inexpensive tool to assess nutritional risk that can be effectively adopted by nurses after specific training, as in this study.

\section{CONCLUSIONS}

This series shows that the NRS is a strong independent predictor of early postoperative morbidity after major open urological surgery. Its usefulness seems more relevant than that of other tools routinely used for preoperative assessment, such as the Charlson comorbidity index and ASA score. Based on these findings assessing nutritional status should be encouraged to stratify perioperative risk. In addition, since malnutrition is a modifiable factor, future studies should investigate the hypothesis that immunonutrition or other forms of preoperative nutritional support can reverse malnutrition and decrease postoperative morbidity.

\section{ACKNOWLEDGMENTS}

Anne-Sophie Legris assisted with the study.

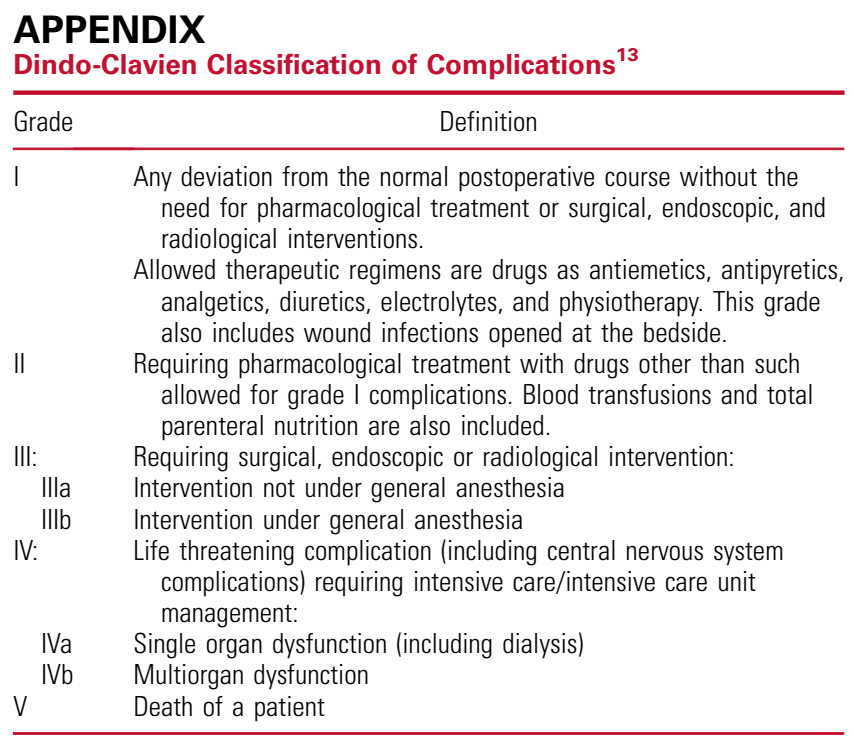

Example: in this therapy based classification a complication such as lymphocele is classified as grade Illa if it is treated with computerized tomography guided drainage puncture, and as IIIb if it requires laparoscopic fenestration.

\section{REFERENCES}

1. Kondrup J, Allison SP, Elia $M$ et al: ESPEN guidelines for nutrition screening 2002. Clin Nutr 2003; 22: 415

2. Schiesser M, Muller $S$, Kirchhoff $P$ et al: Assessment of a novel screening score for nutritional risk in predicting complications in gastro-intestinal surgery. Clin Nutr 2008; 27: 565.

3. Weimann A, Braga M, Harsanyi $L$ et al: ESPEN Guidelines on Enteral Nutrition: Surgery including organ transplantation. Clin Nutr 2006; 25: 224.

4. Mueller C, Compher $C$ and Ellen DM: A.S.P.E.N. clinical guidelines: nutrition screening, assessment, and intervention in adults. JPEN J Parenter Enteral Nutr 2011; 35: 16.

5. Bozzetti F, Gianotti L, Braga M et al: Postoperative complications in gastrointestinal cancer patients: the joint role of the nutritional status and the nutritional support. Clin Nutr 2007; 26: 698.

6. Sorensen J, Kondrup J, Prokopowicz J et al: Euro00PS: an international, multicentre study to implement nutritional risk screening and evaluate clinical outcome. Clin Nutr 2008; 27: 340.
7. Karl A, Rittler P, Buchner A et al: Prospective assessment of malnutrition in urologic patients. Urology 2009; 73: 1072

8. Gregg JR, Cookson MS, Phillips S et al: Effect of preoperative nutritional deficiency on mortality after radical cystectomy for bladder cancer. J Urol 2011; 185: 90.

9. Morgan TM, Tang D, Stratton KL et al: Preoperative nutritional status is an important predictor of survival in patients undergoing surgery for renal cell carcinoma. Eur Urol 2011; 59: 923.

10. Akbarshahi $H$, Andersson $B$, Norden $M$ et al: Perioperative nutrition in elective gastrointestinal surgery-potential for improvement? Dig Surg 2008; 25: 165

11. Cerantola $\mathrm{Y}$, Hubner $\mathrm{M}$, Grass $\mathrm{F}$ et al: Immunonutrition in gastrointestinal surgery. Br J Surg 2011; 98: 37

12. Cerantola $Y$ and Jichlinski P: Quality assessment in urology. Rev Med Suisse 2011; 7: 2382.

13. Dindo D, Demartines N and Clavien PA: Classification of surgical complications: a new proposal with evaluation in a cohort of 6336 patients and results of a survey. Ann Surg 2004; 240: 205.

14. Collett D: Modelling Binary Data, 2nd ed London: Chapman \& Hall 2003.

15. Karl A, Staehler M, Bauer R et al: Malnutrition and clinical outcome in urological patients. Eur $\mathrm{J}$ Med Res 2011; 16: 469.

16. Roth B, Birkhauser FD, Zehnder $P$ et al: Parenteral nutrition does not improve postoperative recovery from radical cystectomy: results of a prospective randomised trial. Eur Urol 2013; 63: 475.

17. Correia Ml and Waitzberg DL: The impact of malnutrition on morbidity, mortality, length of hospital stay and costs evaluated through a multivariate model analysis. Clin Nutr 2003; 22: 235.

18. Martin RC 2nd, Brennan MF and Jaques DP: Quality of complication reporting in the surgical literature. Ann Surg 2002; 235: 803.

19. Donat SM: Standards for surgical complication reporting in urologic oncology: time for a change. Urology 2007; 69: 221. 
20. Dindo D, Hahnloser D and Clavien PA: Quality assessment in surgery: riding a lame horse. Ann Surg 2010; 251: 766.

21. Kurosawa $S$ and Kato $M$ : Anesthetics, immune cells, and immune responses. J Anesth 2008; 22: 263.

22. Kehlet $\mathrm{H}$ : Multimodal approach to control postoperative pathophysiology and rehabilitation. $\mathrm{Br}$ J Anaesth 1997; 78: 606.

23. Alves A, Panis $Y$, Mathieu $P$ et al: Postoperative mortality and morbidity in French patients undergoing colorectal surgery: results of a prospective multicenter study. Arch Surg 2005; 140: 278 .

24. Malone DL, Genuit T, Tracy JK et al: Surgical site infections: reanalysis of risk factors. J Surg Res 2002; 103: 89.

25. Kondrup J, Rasmussen $\mathrm{HH}$, Hamberg 0 et al: Nutritional risk screening (NRS 2002): a new method based on an analysis of controlled clinical trials. Clin Nutr 2003; 22: 321.

26. Cerantola $Y$, Grass F, Cristaudi A et al: Perioperative nutrition in abdominal surgery: recommendations and reality. Gastroenterol Res Pract 2011; 2011: 739347.

27. Gianotti L, Braga M, Nespoli $L$ et al: A randomized controlled trial of preoperative oral supplementation with a specialized diet in patients with gastrointestinal cancer. Gastroenterology 2002; 122: 1763.

28. Stratton RJ and Elia M: Who benefits from nutritional support: what is the evidence? Eur $\mathrm{J}$ Gastroenterol Hepatol 2007; 19: 353. 